\title{
Resting-State Glucose Metabolism Level Is Associated with the Regional Pattern of Amyloid Pathology in Alzheimer's Disease
}

\author{
Jonghan Shin, ${ }^{1,2}$ Wai Tsui,, ${ }^{2}$ Yi Li, ${ }^{2}$ Sang-Yoon Lee, ${ }^{1}$ Seog Ju Kim,, ${ }^{1}$ Seong-Jin Cho, ${ }^{1}$ \\ Young-Bo Kim, ${ }^{1}$ and Mony J. de Leon ${ }^{2,3}$ \\ ${ }^{1}$ Neuroscience Research Institute and Department of Psychiatry, Gachon University of Medicine and Science, \\ Incheon 405-760, Republic of Korea \\ ${ }^{2}$ Department of Psychiatry, New York University School of Medicine, New York, NY 10016, USA \\ ${ }^{3}$ Nathan Kline Institute, Orangeburg, NY 10962, USA \\ Correspondence should be addressed to Jonghan Shin, jhshin@gachon.ac.kr
}

Received 1 October 2010; Accepted 3 January 2011

Academic Editor: Alexander Drzezga

Copyright () 2011 Jonghan Shin et al. This is an open access article distributed under the Creative Commons Attribution License, which permits unrestricted use, distribution, and reproduction in any medium, provided the original work is properly cited.

\begin{abstract}
It has been suggested that glucose metabolism within the brain's default network is directly associated with—and may even causethe amyloid pathology of Alzheimer's disease (AD). Here we performed 2- $\left[{ }^{18} \mathrm{~F}\right]$ fluoro-2-deoxy-D-glucose (FDG) and $\left[{ }^{11} \mathrm{C}\right]$-labeled Pittsburgh Compound B (PIB) positron emission tomography (PET) on cognitively normal elderly subjects and on AD patients and conducted quantitative regional analysis of FDG- and PIB-PET images using an automated region of interest technique. We confirmed that resting glucose metabolism within the posterior components of the brain's default network is high in normal elderly subjects and low in $\mathrm{AD}$ patients, which is partially in agreement with the regional pattern of PIB uptake within the default network of $\mathrm{AD}$ patients. However, in several regions outside the default network, glucose metabolism was high in normal elderly subjects but was not depressed in AD patients, who exhibited significantly increased PIB uptakes in these regions. In contrast, the level of resting glucose metabolism in the default network and in regions outside the default network in normal elderly subjects was significantly correlated with the level of regional PIB uptake in AD patients. These results are discussed with experimental evidence suggesting that beta amyloid production and amyloid precursor protein regulation are dependent on neuronal activity.
\end{abstract}

\section{Introduction}

The concept of a default mode network, an interconnected set of brain regions (the frontal, parietal, posterior cingulate, lateral, and medial temporal cortices) that is active when the brain is in a resting state and deactivated during focused mental tasks, was first proposed in 2001 [1]. But it rapidly became the target of much research on Alzheimer's disease $(\mathrm{AD})$, the most common form of dementia and a challenging global public health problem [2].

The earliest evidence that the default network is disrupted in $\mathrm{AD}$ was provided by positron emission tomography (PET) studies of resting glucose metabolism using 2 - $\left[{ }^{18} \mathrm{~F}\right]$ fluoro-2-deoxy-D-glucose (FDG). AD patients show a specific anatomic pattern of reduced glucose metabolism relative to age-matched healthy controls [3-10]. This pattern corresponds with the posterior components of the default network (the posterior cingulate cortex (PCC), inferior parietal lobule (IP), and lateral temporal cortex (LTC)) [11].

Recently, molecular imaging using $\left[{ }^{11} \mathrm{C}\right]$-labeled Pittsburgh Compound B (PIB) revealed the amyloid pathology preferentially accumulates in the default network $[2,9-18]$. Therefore, it was suggested that metabolic activity in the default network may be associated with-or even cause - the pathology of $\mathrm{AD}[2,9,11]$.

However, there has been no systematic quantitative investigation of resting glucose metabolism and amyloid pathology within default network regions as contrasted with regions outside the default network. Here, we conducted FDG- and PIB-PET imaging of cognitively normal elderly subjects and $\mathrm{AD}$ patients. We then compared their glucose metabolism and amyloid pathology using quantitative regional analysis of FDG- and PIB-PET images via an automated region of interest (ROI) technique. We also compared 
TABLe 1: Demographic information.

\begin{tabular}{lcc}
\hline Subjects & Control & AD patients \\
\hline Number & 14 & 7 \\
Age (yr) & $67 \pm 4$ & $70 \pm 7$ \\
Sex (F/M) & $9 / 5$ & $5 / 2$ \\
Handness (right/left) & $14 / 0$ & $7 / 0$ \\
MMSE-K & $29 \pm 1$ & $18 \pm 3$ \\
CDR & 0 & $1.6 \pm 0.8$ \\
\hline
\end{tabular}

Mean \pm SD values shown.

$\mathrm{AD}=$ Alzheimer disease MMSE-K = Korean version of Mini Mental State Examination; CDR = Clinical Dementia Rating.

ApoE4 status of subjects was not available.

FDG- and PIB-PET patterns in the default network regions with those in regions outside the default network.

\section{Materials and Methods}

2.1. Subjects. Twenty-one subjects were included in this study. Written informed consent was obtained after a detailed explanation of the procedure, which was reviewed by the Institute Review Board of the Gachon University of Medicine and Science, Incheon, South Korea. None of the subjects had a previous history of other neurological, medical, or psychiatric conditions. All subjects received neurological, psychiatric, and neuropsychological evaluations, PIB- and FDG-PET scans and a magnetic resonance imaging (MRI) scan in addition to routine laboratory tests. Brain MRI scans were used to confirm that territorial cerebral infarctions, brain tumors, and other structural lesions were absent. AD patients were identified using the standard diagnostic criteria of memory impairment, impairment in at least one other cognitive domain, gradual onset and progressive decline, and impaired occupational or social functioning or both [19]. Control subjects were cognitively normal for age and did not meet the criteria for mild cognitive impairment or AD. Seven subjects (five females and two males) met the diagnostic criteria for dementia of the Alzheimer type, and 14 subjects (nine females and five males) were cognitively normal (controls). The mean age and standard deviation (SD) was $70 \pm 7$ years for $\mathrm{AD}$ subjects and $67 \pm 4$ years for control subjects. The age difference between the two groups was not statistically significant $(P>.05)$. The results of mini mental state examinations, a measure of global cognitive function, were (mean \pm SD) $18 \pm 3$ for AD subjects and 29 \pm 1 for the controls $(P<.001)$. Clinical dementia ratings were zero for control subjects and $1.6 \pm 0.8($ mean \pm SD) for $\mathrm{AD}$ patients. Demographic information is summarized in Table 1.

2.2. PET Imaging. $\left[{ }^{18} \mathrm{~F}\right] \mathrm{FDG}$ was prepared at a very high specific activity as described previously [20]. For FDGPET, PET scans of $20 \mathrm{~min}$ were acquired $40 \mathrm{~min}$ after an intravenous injection of $4.8 \mathrm{MBq} / \mathrm{kg}$ of FDG. Participants had fasted for at least $6 \mathrm{~h}$ before the FDG-PET scan. To minimize external stimuli during the radiotracer uptake period, participants remained in a dimly lit room with their eyes closed. The PET imaging probe for visualizing amyloid plaques (PIB) was prepared according to published protocols [21]. For PIB-PET, $20 \mathrm{~min}$ PET scans were acquired $40 \mathrm{~min}$ after an intravenous injection of $9.6 \mathrm{MBq} / \mathrm{kg}$ of PIB on a different day to that on which FDG-PET scans were obtained. Our scanning protocol (i.e., $20 \mathrm{~min}$ static scan $40 \mathrm{~min}$ after injection for both FDG and PIB) has been previously validated by Lopresti et al. [22] for PIB and Mosconi et al. [23] for FDG, respectively. All brain scans were performed using a Biograph 6 Hi-Rez scanner (Siemens-CTI, Knoxville, $\mathrm{TN}$ ) in three-dimensional (3D) acquisition mode after administration of an intravenous injection of a bolus of PET tracer. The Biograph 6 is a commercial PET/CT (computed tomography) human whole-body scanner with an electrical field of view $585 \mathrm{~mm}$ in width and $162 \mathrm{~mm}$ in axial length. The Biograph 6 uses Hi-Rez PET scan modules to achieve a very fine spatial resolution of $4-5 \mathrm{~mm}$, and performs attenuation-correction transmission measurements of the emission scans using X-ray CT. An X-ray source rotation speed of $0.8 \mathrm{~s}$ at $130 \mathrm{kVp}$ with a tube current of $240 \mathrm{~mA}$ was used to generate the X-ray CT scan. All PET scans were decay-corrected and reconstructed using an iterative reconstruction algorithm based on ordered-subset expectation maximization with scatter correction and measured attenuation correction. OSEM images underwent $5.5 \mathrm{~mm}$ full-width-at-half-maximum Gaussian postsmoothing.

2.3. PET Data Quantification. $\left[{ }^{11} \mathrm{C}\right] \mathrm{PIB}$ quantification was performed using a relative standard uptake value (SUVR; evaluated at equilibrium and normalized for the cerebellum gray matter) of 40-60 min. This analytic method has been validated previously [22]. Of several different PIB quantification methods, the late-scan reference method has shown a large size effect [22]. [ $\left.{ }^{18} \mathrm{~F}\right] \mathrm{FDG}$ quantification was done using an SUVR normalized for cerebellum gray matter of 40$60 \mathrm{~min}$. This quantification protocol has been validated previously [23]. The gray matter of the cerebellum was used as the reference region for both PIB and FDG analyses because the cerebellum is minimally affected by reductions in glucose metabolism [3, 6] and amyloid pathology [24, 25] in AD.

2.4. MRI. Anatomical brain MRI scans were acquired using a $1.5 \mathrm{~T}$ Siemens Avanto system (Erlangen, Germany) with a 3D T1-MPRAGE sequence as follows: $\mathrm{TR}=1160 \mathrm{~ms}$, $\mathrm{TE}=$ $4.24 \mathrm{~ms}, \mathrm{TI}=600 \mathrm{~ms}, \mathrm{FA}=15^{\circ}, \mathrm{BW}=180 \mathrm{~Hz} / \mathrm{Px}$, matrix size $=512 \times 512$, number of slices $=192$, slice thickness $=1 \mathrm{~mm}$, $\mathrm{FOV}=25.6 \times 25.6 \mathrm{~cm}^{2}$, and NEX $=1$.

2.5. ROIs. All image processing and data analyses were performed blind to clinical diagnoses. Coregistering of PIBand FDG-PET images with the corresponding MRI images was performed using statistical parameter mapping software (SPM5; Wellcome Department of Cognitive Neurology, Institute of Neurology, London, UK), which was implemented using Matlab 6.5 (MathWorks Inc., Sherborn, MA).

The coregistered FDG- and PIB-PET images were transferred to a Sun Sparc workstation (Sun Microsystems, Mountain View, CA) for automated ROI analysis. An MRIbased automated ROI technique validated by a manual ROI 

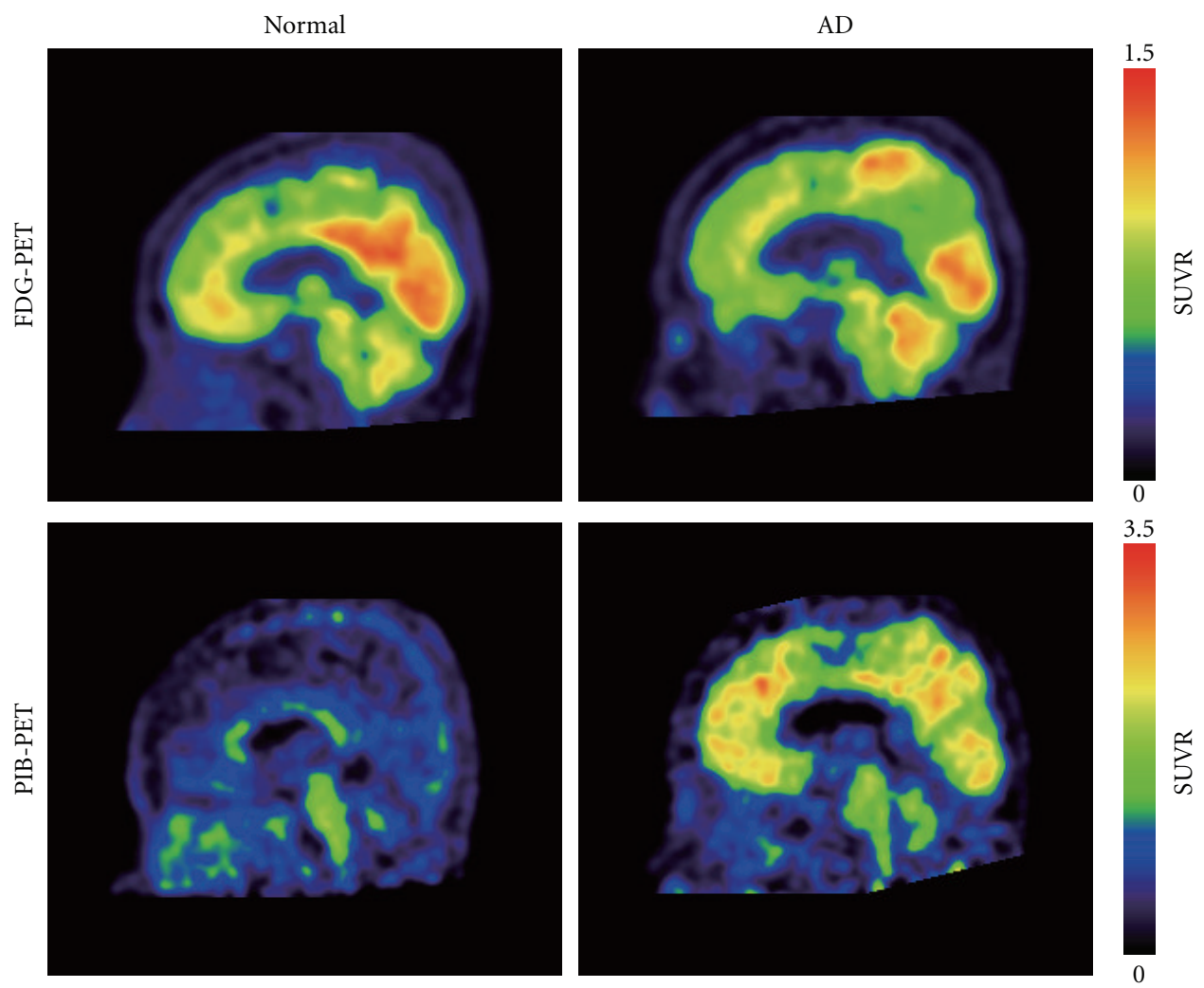

Figure 1: FDG and PIB-PET images of a representative normal elderly subject and an Alzheimer's disease (AD) patient. (a) Positron emission tomography (PET) imaging of FDG in a normal 65-year-old man (left upper panel) and a 60-year-old woman with AD (right upper panel). (b) PET imaging of PIB in the same normal subject (left lower panel) and the same AD patient (right lower panel).

technique was used to sample each individual's FDG and PIB images (see [14] for details). Briefly, the template ROI was first developed using seven MRI scans and was then transferred to a coregistered MNI PET template. All PET scans were normalized to the PET template using highorder polynomial transformation [26]. Having saved the spatial normalization parameters, an inverse transformation was applied to morph the ROIs back to the original FDGPET. The standard FDG-ROIs were then transferred to the PIB scan in real space through the coregistration. ROI positioning was verified on the MRI, but no positioning adjustments were made in this project. To maximize gray matter sampling, a probabilistic gray matter template image derived from the SPM software was applied to the template ROI (see [14]). Fifteen MRI and FDG-PET-validated automated ROIs were studied: the IP, PCC, LTC, putamen (PU), visual cortex (VC), occipital cortex (OC), hippocampus (HIP), middle frontal gyrus (MFG), superior temporal gyrus (STG), thalamus (TH), amygdala (AMY), medial temporal cortex (MTC), prefrontal cortex (PFC), orbitofrontal cortex (OFC), and cerebellum.

2.6. Statistical Analysis. Independent Student's $t$-tests were used to assess the significance of differences in PIB and FDG means between $\mathrm{AD}$ subjects and controls. We performed ANOVA to compare differences in radiotracer uptake within different brain regions between $\mathrm{AD}$ subjects and controls, which was followed by Tukey's post hoc test. Pearson's correlations were used to assess bivariate relationships (SPSS Version 12, SPSS Inc., Chicago, IL).

\section{Results}

The upper left panel of Figure 1 shows an FDG-PET image of a representative normal elderly subject. The posterior components of the default network (the posterior cingulate cortex and the precuneus) exhibited higher resting glucose metabolism than most other brain regions. However, it should be noted that the high glucose metabolism was not restricted to the default network because the primary visual cortex also exhibited high resting glucose metabolism (Figure 1, upper left panel). The FDG-PET image from a representative $\mathrm{AD}$ patient (Figure 1, upper right panel) shows hypometabolism in the posterior cingulated cortex and the precuneus. In contrast, the visual cortex of the $\mathrm{AD}$ patient did not show hypometabolism (Figure 1, upper right panel). On the other hand, a PIB-PET image from the same AD patient showed increased PIB uptake not only in parts of the default network such as the posterior cingulated cortex and the precuneus, but also in regions outside the default network (e.g., the visual cortex) (Figure 1, lower right panel). These observations show that regions outside the default network may exhibit high resting glucose metabolism and amyloid pathology. 
TABle 2: 2- $\left[{ }^{18} \mathrm{~F}\right]$ fluoro-2-deoxy-D-glucose (FDG) and $\left[{ }^{11} \mathrm{C}\right]$-labeled Pittsburgh Compound B (PIB) uptakes in the default network regions and regions outside the default network in Alzheimer's disease (AD) and control subjects.

\begin{tabular}{|c|c|c|c|c|c|}
\hline \multirow{2}{*}{\multicolumn{2}{|c|}{$\begin{array}{c}\text { Subject } \\
\text { Radiotracer }\end{array}$}} & \multicolumn{2}{|c|}{ Control } & \multicolumn{2}{|c|}{$\mathrm{AD}$} \\
\hline & & FDG & PIB & FDG & PIB \\
\hline \multirow{8}{*}{ Default network } & MFG & $1.20 \pm 0.074$ & $1.09 \pm 0.104$ & $0.96 \pm 0.137^{\dagger \dagger}$ & $1.92 \pm 0.229^{* *}$ \\
\hline & MTC & $0.81 \pm 0.041$ & $1.06 \pm 0.084$ & $0.74 \pm 0.069^{\dagger}$ & $1.16 \pm 0.126^{*}$ \\
\hline & LTC & $0.98 \pm 0.033$ & $1.11 \pm 0.099$ & $0.83 \pm 0.120^{\dagger}$ & $1.63 \pm 0.207^{* *}$ \\
\hline & IP & $1.11 \pm 0.045$ & $1.09 \pm 0.078$ & $0.90 \pm 0.123^{\dagger \dagger}$ & $1.84 \pm 0.164^{* *}$ \\
\hline & PCC & $1.21 \pm 0.039$ & $1.10 \pm 0.090$ & $1.00 \pm 0.158^{\dagger}$ & $2.11 \pm 0.198^{* *}$ \\
\hline & PFC & $1.11 \pm 0.042$ & $1.08 \pm 0.152$ & $0.93 \pm 0.115^{\dagger}$ & $1.88 \pm 0.183^{* *}$ \\
\hline & OFC & $1.10 \pm 0.043$ & $1.02 \pm 0.163$ & $0.92 \pm 0.123^{\dagger}$ & $1.74 \pm 0.161^{* *}$ \\
\hline & HIP & $0.82 \pm 0.052$ & $1.03 \pm 0.082$ & $0.76 \pm 0.109$ & $1.03 \pm 0.116$ \\
\hline \multirow{6}{*}{ Nondefault network } & PU & $1.21 \pm 0.080$ & $1.21 \pm 0.194$ & $1.16 \pm 0.118$ & $2.05 \pm 0.350^{* *}$ \\
\hline & STG & $1.04 \pm 0.042$ & $1.13 \pm 0.090$ & $0.89 \pm 0.135^{\dagger}$ & $1.57 \pm 0.216^{*}$ \\
\hline & $\mathrm{TH}$ & $1.19 \pm 0.090$ & $1.29 \pm 0.109$ & $1.08 \pm 0.115^{\dagger}$ & $1.44 \pm 0.250$ \\
\hline & $\mathrm{VC}$ & $1.24 \pm 0.050$ & $1.05 \pm 0.117$ & $1.28 \pm 0.131$ & $1.46 \pm 0.250^{* *}$ \\
\hline & $\mathrm{OC}$ & $1.13 \pm 0.048$ & $1.09 \pm 0.098$ & $1.12 \pm 0.119$ & $1.57 \pm 0.220^{*}$ \\
\hline & AMY & $0.72 \pm 0.050$ & $1.00 \pm 0.096$ & $0.69 \pm 0.062$ & $1.06 \pm 0.109$ \\
\hline
\end{tabular}

Mean \pm SD.

${ }^{\dagger} P<.05$ versus FDG control, two-tailed $t$ test.

${ }^{\dagger \dagger} P<.001$ versus FDG control.

$* P<.05$ versus PIB control, two-tailed $t$ test.

$* * P<.001$ versus. PIB control.

MFG: middle frontal gyrus, MTC: medial temporal cortex, LTC: lateral temporal cortex, IP: inferior parietal lobe, PCC: posterior cingulate cortex, PFC: prefrontal cortex, OFC: orbitofrontal cortex, HIP: hippocampus, PU: putamen, STG: superior temporal gyrus, TH: thalamus, VC: visual cortex, OC: occipital cortex, AMY: amygdala.

To compare resting glucose metabolism and PIB uptake within the default network regions with those in regions outside the default network, we performed quantitative regional analysis of FDG- and PIB-PET images using an automated ROI technique.

3.1. Quantitative Regional Analysis of FDG-PET Images. FDG uptakes by the default network regions and regions outside the default network in $\mathrm{AD}$ and control subjects are shown in Table 2. Comparison between the AD and control groups revealed that (1) in the default network, the resting glucose metabolism of $\mathrm{AD}$ patients was significantly less than that of the controls in the MFG, MTC, LTC, IP, PCC, PFC, and OFC; (2) in the default network, resting glucose metabolism was statistically weakly reduced in the HIP of $\mathrm{AD}$ patients compared with controls (AD patients, $0.76 \pm 0.109$; controls, $0.82 \pm 0.052 ; P=.076) ;(3)$ in the nondefault network, the resting glucose metabolism of $\mathrm{AD}$ patients was significantly less than that of the controls in the STG and TH; (4) in the nondefault network, resting glucose metabolism did not differ significantly between $\mathrm{AD}$ patients and controls in the PU, VC, OC, and AMY.

Figure 2 shows the mean and SE of SUVRs for FDG-PET for the default and nondefault networks of normal elderly subjects and $\mathrm{AD}$ patients. In normal elderly subjects, the regions of the brain can be classed into two groups based on resting glucose metabolism: regions with high resting glucose metabolism (the MFG, LTC, IP, PCC, PFC, and OFC in the default network and the PU, STG, TH, VC, and OC in the nondefault network), and regions with low resting glucose metabolism (the MTC and the HIP in the default network and the AMY in the nondefault network).

3.2. Quantitative Regional Analysis of PIB-PET Images. PIB uptakes for various brain regions of the $\mathrm{AD}$ and control groups are shown in Table 2. Comparison of the $\mathrm{AD}$ and control groups revealed that (1) in the default network, PIB uptake of $\mathrm{AD}$ patients was significantly greater than that of the controls in the MFG, MTC, LTC, IP, PCC, PFC, and OFC; (2) in the default network, PIB uptake did not differ significantly between AD patients and controls in the HIP; (3) in the nondefault network, PIB uptake of AD patients was significantly greater than that of the controls in the PU, STG, VC, and OC; (4) in the nondefault network, PIB uptake did not differ significantly between $\mathrm{AD}$ patients and controls in the TH and AMY.

Figure 3 shows the mean and SE of SUVRs for PIB-PET for the default and nondefault networks of normal elderly subjects and $\mathrm{AD}$ patients. In $\mathrm{AD}$ patients, the regions of the brain can be classed into two groups based on PIB uptake: regions with high PIB uptake (the MFG, LTC, IP, PCC, PFC, and OFC in the default network and the PU, STG, TH, VC, and $\mathrm{OC}$ in the nondefault network) and regions with low PIB uptake (the MTC and HIP in the default network and the AMY in the nondefault network).

3.3. Comparison of PIB and FDG-PET Regional Patterns. Table 3 shows patterns of resting glucose metabolism and amyloid deposition for the default and nondefault networks. In the default network of $\mathrm{AD}$ patients, the pattern 


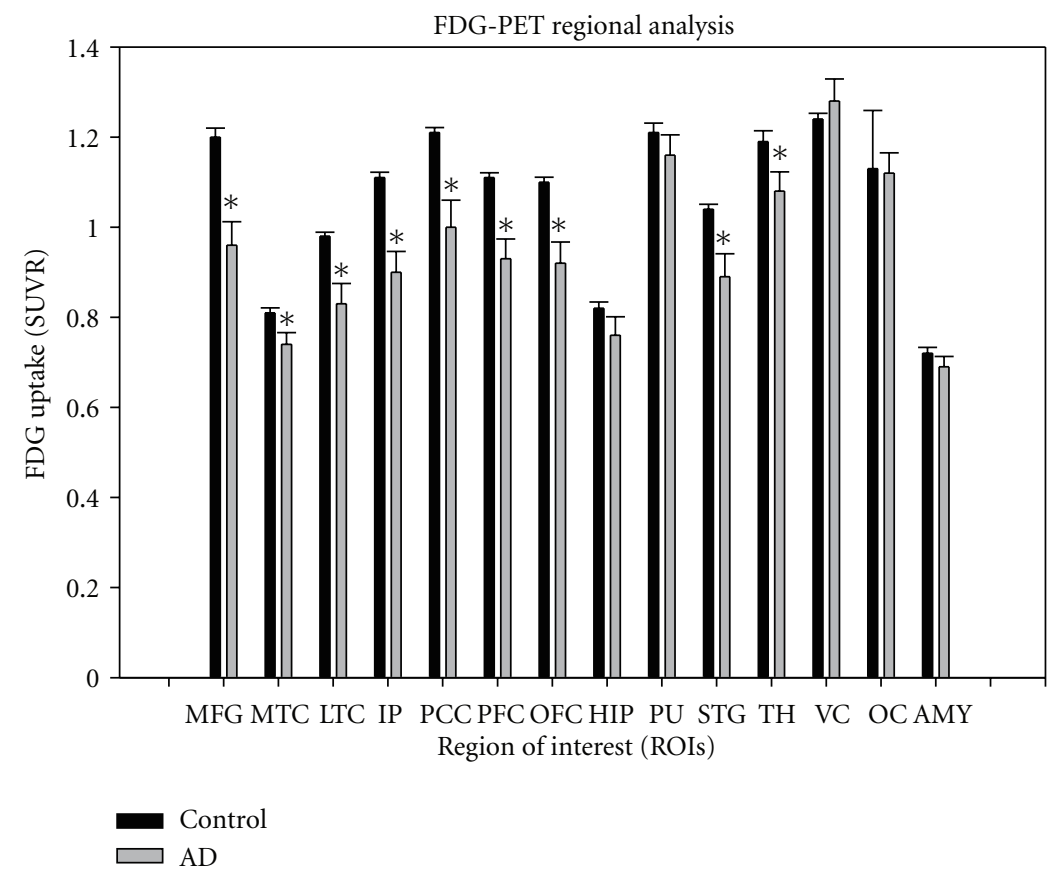

FIGURE 2: Regional FDG uptakes in normal control subjects and Alzheimer's disease (AD) patients. Mean and SE of relative standardized uptake values (SUVRs) for FDG-PET in various brain regions in normal (black bars) and AD subjects (gray bars). MFG: middle frontal gyrus, MTC: medial temporal cortex, LTC: lateral temporal cortex, IP: inferior parietal lobe, PCC: posterior cingulate cortex, PFC: prefrontal cortex, OFC: orbitofrontal cortex, HIP: hippocampus, PU: putamen, STG: superior temporal gyrus, TH: thalamus, VC: visual cortex, OC: occipital cortex, AMY: amygdala. ${ }^{*} P<.05$, two-tailed $t$ test.

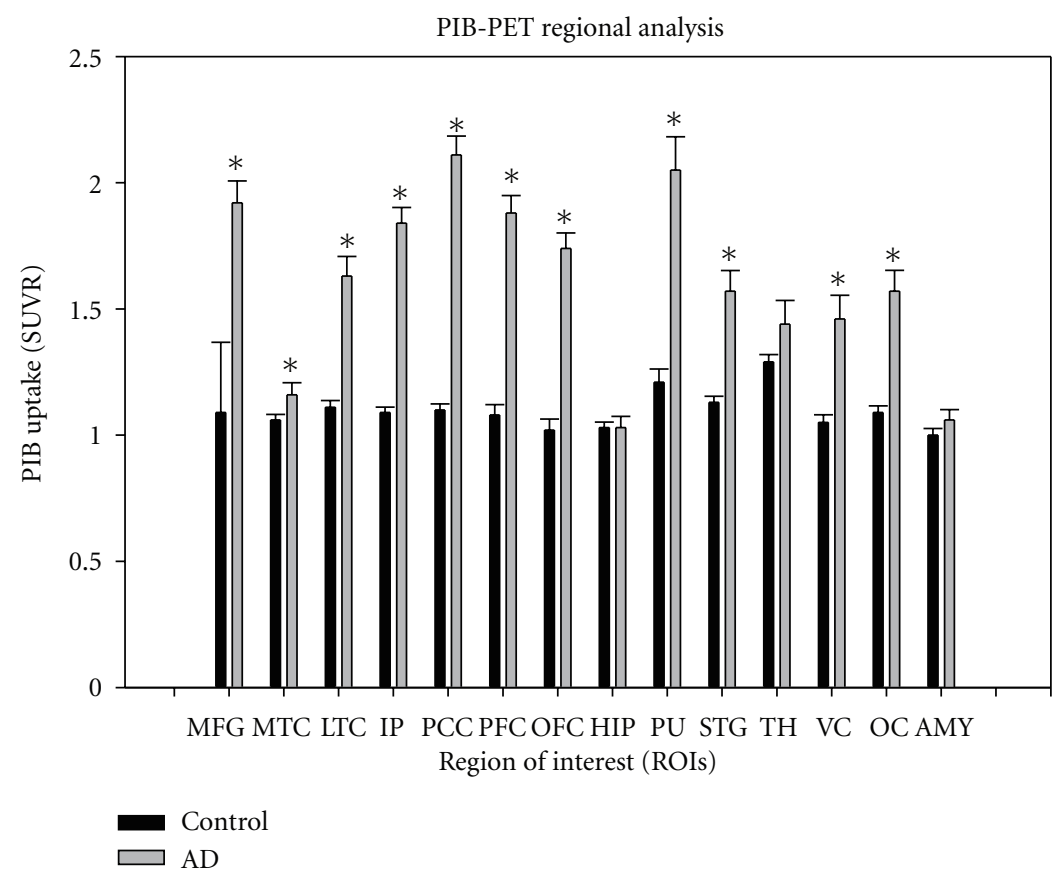

FIGURE 3: Regional PIB uptakes in normal control subjects and Alzheimer's disease (AD) patients. Mean and SE of SUVRs for PIB-PET in various brain regions in normal (black bars) and AD subjects (gray bars). MFG: middle frontal gyrus, MTC: medial temporal cortex, LTC: lateral temporal cortex, IP: inferior parietal lobe, PCC: posterior cingulate cortex, PFC: prefrontal cortex, OFC: orbitofrontal cortex, HIP: hippocampus, PU: putamen, STG: superior temporal gyrus, TH: thalamus, VC: visual cortex, OC: occipital cortex, AMY: amygdala. $* P<.05$, two-tailed $t$ test. 


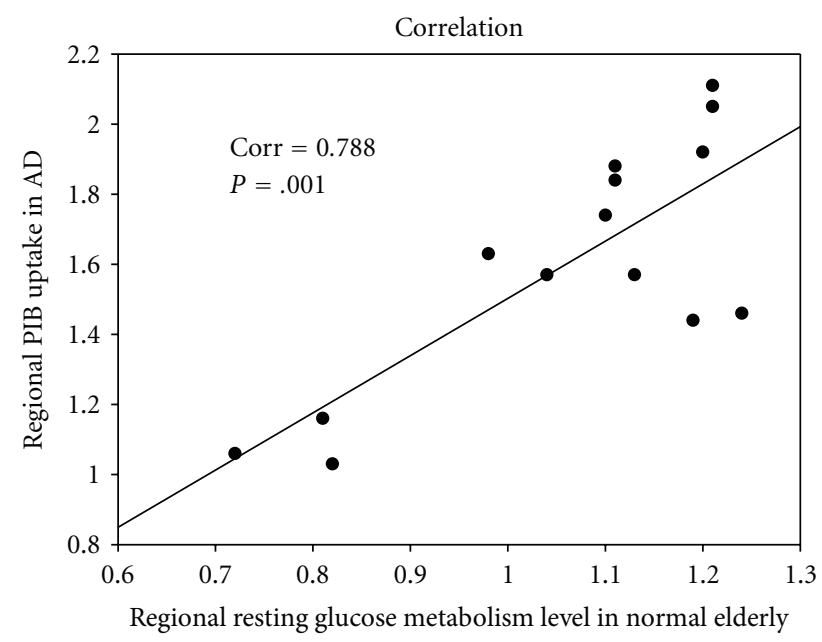

FIGURE 4: The relationship between resting glucose metabolism levels and PIB uptakes. The correlation between regional resting glucose metabolism in normal elderly subjects and regional PIB uptake in Alzheimer's disease (AD) patients from various different brain areas is highly significant $(0.788, P=.001)$. This correlation demonstrates that brain regions with high resting glucose metabolism in normal elderly subjects have high $\mathrm{PIB}$ uptakes in $\mathrm{AD}$ patients and that brain regions with low resting glucose metabolism have low PIB uptakes in AD patients. Most importantly, it should be noted that resting glucose metabolism level and PIB uptake are correlated not only in the brain's default network regions but also in brain regions outside the default network.

of resting glucose metabolism was associated with the pattern of hypometabolism and the pattern of PIB uptake. This result is consistent with previous reports. However, nondefault network regions such as the PU and VC showed no hypometabolism but had high PIB uptake. Therefore, the pattern of hypometabolism does not correspond with that of PIB uptake in $\mathrm{AD}$ patients.

In contrast, the pattern of resting glucose metabolism corresponded with the pattern of PIB uptake in nondefault network regions. Therefore, we conducted correlation analysis between regional resting FDG SUVR values in normal elderly subjects and regional PIB SUVR values in $\mathrm{AD}$ patients, and observed a highly significant correlation (Figure 4) $(0.788, P=.001)$. This result suggests that brain regions with high resting glucose metabolism may accelerate the formation of amyloid pathology associated with $\mathrm{AD}$.

\section{Discussion}

To our knowledge, this is the first quantitative study on the relationship between resting glucose metabolism and amyloid pathology in default network regions as well as in regions outside the default network. In addition to confirming the well-known regional associations between glucose metabolism and PIB uptake in the brain's default network, this study investigated whether brain regions outside the default network exhibit the same relationships. Surprisingly, we found that whereas resting glucose metabolism level was associated with the pattern of PIB uptake in the nondefault network, the pattern of glucose hypometabolism did not match the amyloid pathology pattern in the nondefault network. Taken together, we found a statistically significant correlation between the level of resting glucose metabolism in normal elderly subjects and PIB uptake in AD patients in the default and nondefault network regions.

That the PU and VC exhibited high PIB uptake but insignificant glucose hypometabolism, suggests that amyloid plaque accumulation per se does not result in glucose hypometabolism in $\mathrm{AD}$. For the same reason, it is also inferred that glucose hypometabolism per se does not result in amyloid plaque accumulation (see Figures 2 and 3 ). If low glucose metabolism is not associated with amyloid plaque accumulation, what is the association between high glucose metabolism and amyloid plaque accumulation? It has been suggested that high baseline levels of glucose metabolism in the default network may be conducive to the formation of amyloid pathology associated with $\mathrm{AD}[2,9,11]$. Our results show that this proposal may be generalized as follows. Regardless of whether a brain region belongs to the default network or not, the resting glucose metabolism level within a region in healthy subjects is associated with the level of amyloid pathology in that region in $\mathrm{AD}$ patients. The statistically significant correlation between the regional resting glucose metabolism level in normal elderly subjects and regional PIB uptake values in $\mathrm{AD}$ patients in both default network regions and nondefault network regions supports this generalization (see Figure 4).

Histological studies have demonstrated that amyloid plaque deposition is prominent in the neocortical regions but is not especially prominent in the MTC and the HIP [27]. To date, all PIB-PET imaging studies have consistently confirmed this histological feature of amyloid plaque deposition in that PIB uptake is high in the neocortical regions but is minimal in the HIP and the MTC $[11,13,14,16]$. If so, what kind of mechanism is responsible for this unique feature of the regional pattern of amyloid plaque deposition? 
TABLE 3: Comparison of the patterns of resting glucose metabolism, glucose hypometabolism, and amyloid deposition in the default and nondefault networks.

\begin{tabular}{|c|c|c|c|c|}
\hline Region & & Resting metabolism level & Hypometabolism & Amyloid accumulation \\
\hline \multirow{8}{*}{ Default network } & MFG & + & + & + \\
\hline & MTC & - & $-1+$ & $-/+$ \\
\hline & LTC & + & + & + \\
\hline & IP & + & + & + \\
\hline & PCC & + & + & + \\
\hline & PFC & + & + & + \\
\hline & OFC & + & + & + \\
\hline & HIP & - & $-1+$ & - \\
\hline \multirow{6}{*}{ Nondefault network } & $\mathrm{PU}$ & + & - & + \\
\hline & STG & + & + & + \\
\hline & $\mathrm{TH}$ & + & + & $-1+$ \\
\hline & $\mathrm{VC}$ & + & - & + \\
\hline & OC & + & - & + \\
\hline & AMY & - & - & - \\
\hline
\end{tabular}

-, low effect; +, high effect; -/+, minimal effect.

MFG: middle frontal gyrus, MTC: medial temporal cortex, LTC: lateral temporal cortex, IP: inferior parietal lobe, PCC: posterior cingulate cortex, PFC: prefrontal cortex, OFC: orbitofrontal cortex, HIP: hippocampus, PU: putamen, STG: superior temporal gyrus, TH: thalamus, VC: visual cortex, OC: occipital cortex, AMY: amygdala.

Several lines of evidence suggest that beta amyloid production and amyloid precursor protein regulation are dependent on neuronal activity [28-32]. Beta amyloid peptide $(\mathrm{A} \beta)$ is secreted from healthy neurons in response to neuronal activity, and $A \beta$, in turn, downregulates excitatory synaptic transmission [29]. This negative feedback loop, in which neuronal activity promotes $A \beta$ production, which decreases synaptic activity, represents a homeostatic mechanism for controlling the level of neuronal activity [30]. It was suggested that the regulatory feedback loop between neuronal activity and $\mathrm{A} \beta$ production is impaired in $\mathrm{AD}$ patients, resulting in unchecked accumulation of $A \beta$ and neurotoxicity [29].

The energy metabolism of the adult mammalian brain is almost entirely dependent on glucose and the majority of the glucose taken up by the brain is used for maintenance of membrane potentials and electrical activity [33]. Because neuronal activity is closely associated with energy metabolism, measurements of cerebral glucose metabolism can be used to gain insights into the neuronal activity of all of the neuroanatomically defined regions of the brain $[34,35]$. Sensors of glucose metabolism are necessary to make adaptive changes to variations in glucose metabolism. It has been proposed that the adenosine monophosphateactivated protein kinase (AMPK) signaling complex is a glucose metabolism sensor and that it may be involved in neuroprotective processes (see [36] for a review). In addition, a recent animal study provided evidence that AMPK is a key regulator of AD-related pathology [37]. Taken together, given the regulatory feedback loop between neuronal activity and $\mathrm{A} \beta$ production $[29,30]$, it is possible that high resting glucose metabolism, as reflected by FDG-PET, may accelerate the formation of amyloid pathology in $\mathrm{AD}$. If this is the case, the low level of resting glucose metabolism in the HIP and the
MTC (see Figure 2) relative to that in the neocortical regions may explain why we observed minimal PIB uptake in the HIP and the MTC of AD patients (see Figure 3).

On the other hand, recently the relationship between gray matter atrophy and amyloid deposition in $\mathrm{AD}$ was investigated using volumetric magnetic resonance and PIBPET [38]. This study found that significant correlations between atrophy and increased PIB uptake were found in the hippocampal $(r=-0.54)$ and amygdalar ROIs $(r=-0.40)$ but not in the frontal, temporal, posterior cingulate/retrosplenial, insular, and caudate ROIs ( $r$ between 0.04 and 0.25 ). Therefore, these results suggest that the hippocampus and amygdala might be highly susceptible to amyloid toxicity, whereas neocortical areas might be more resilient. Based on this suggestion, an alternative explanation for our present findings is that different brain areas may be differentially susceptible for amyloid toxicity.

In conclusion, within the default network, resting glucose metabolism level in healthy subjects, glucose hypometabolism in $\mathrm{AD}$ patients, and amyloid pathology in $\mathrm{AD}$ patients are coincident (see Table 3 ). Therefore, it is difficult to determine whether high resting glucose metabolism or decreased metabolism is associated with amyloid pathology. Our quantitative regional analysis demonstrates that high resting glucose metabolic activity, rather than glucose hypometabolism, is associated with the pattern of beta amyloid plaque (or PIB uptake) in $\mathrm{AD}$ (see Table 3 and Figure 4). However, it should be also noted that the patient population in the current group is utterly small. Therefore, the results based on this small sample are very preliminary and only give a first hint that high resting metabolism may be associated with later amyloid deposition. These results need to be longitudinally confirmed in larger samples before they can be transferred to the general population. 


\section{Acknowledgments}

This research was supported by the Gil Foundation (Incheon, Republic of Korea) and NIH Grants AG13616, AG032554, AG12101, AG036502, AG035137, AG22374, and AG08051.

\section{References}

[1] D. A. Gusnard and M. E. Raichle, "Searching for a baseline: functional imaging and the resting human brain," Nature Reviews Neuroscience, vol. 2, no. 10, pp. 685-694, 2001.

[2] A. G. Vlassenko, S. N. Vaishnavi, L. Couture et al., "Spatial correlation between brain aerobic glycolysis and amyloid$\beta$ (A $\beta)$ deposition," Proceedings of the National Academy of Sciences of the United States of America, vol. 107, no. 41, pp. 17763-17767, 2010.

[3] D. F. Benson, D. E. Kuhl, R. A. Hawkins, M. E. Phelps, J. L. Cummings, and S. Y. Tsai, "The fluorodeoxyglucose F scan in Alzheimer's disease and multi-infarct dementia," Archives of Neurology, vol. 40, no. 12, pp. 711-714, 1983.

[4] A. Kumar, M. B. Schapiro, C. Grady et al., "High-resolution PET studies in Alzheimer's disease," Neuropsychopharmacology, vol. 4, no. 1, pp. 35-46, 1991.

[5] K. Herholz, "FDG PET and differential diagnosis of dementia," Alzheimer Disease and Associated Disorders, vol. 9, no. 1, pp. 616, 1995.

[6] S. Minoshima, B. Giordani, S. Berent, K. A. Frey, N. L. Foster, and D. E. Kuhl, "Metabolic reduction in the posterior cingulate cortex in very early Alzheimer's disease," Annals of Neurology, vol. 42, no. 1, pp. 85-94, 1997.

[7] M. J. de Leon, A. Convit, O. T. Wolf et al., "Prediction of cognitive decline in normal elderly subjects with 2[(18)F] fluoro-2-deoxy-D-glucose/positron-emission tomography (FDG/PET)," Proceedings of the National Academy of Sciences of the United States of America, vol. 98, no. 19, pp. 10966-10971, 2001.

[8] G. E. Alexander, K. Chen, P. Pietrini, S. I. Rapoport, and E. M. Reiman, "Longitudinal PET evaluation of cerebral metabolic decline in dementia: a potential outcome measure in Alzheimer's disease treatment studies," American Journal of Psychiatry, vol. 159, no. 5, pp. 738-745, 2002.

[9] Y. I. Sheline, J. R. Andrews-Hanna, M. E. Raichle, A. Z. Snyder et al., "Amyloid plaques disrupt resting state default mode network connectivity in cognitively normal elderly," Biological Psychiatry, vol. 67, no. 6, pp. 584-587, 2010.

[10] J. Shin, S. Y. Lee, S. J. Kim, S. H. Kim, S. J. Cho, and Y. B. Kim, "Voxel-based analysis of Alzheimer's disease PET imaging using a triplet of radiotracers: PIB, FDDNP, and FDG," NeuroImage, vol. 52, no. 2, pp. 488-496, 2010.

[11] R. L. Buckner, A. Z. Snyder, B. J. Shannon et al., "Molecular, structural, and functional characterization of Alzheimer's disease: evidence for a relationship between default activity, amyloid, and memory," Journal of Neuroscience, vol. 25, no. 34, pp. 7709-7717, 2005.

[12] W. E. Klunk, H. Engler, A. Nordberg et al., "Imaging brain amyloid in Alzheimer's disease with Pittsburgh compound-B," Annals of Neurology, vol. 55, no. 3, pp. 306-319, 2004.

[13] N. M. Kemppainen, S. Aalto, I. A. Wilson et al., "Voxel-based analysis of PET amyloid ligand [11C]PIB uptake in Alzheimer disease," Neurology, vol. 67, no. 9, pp. 1575-1580, 2006.

[14] Y. Li, J. O. Rinne, L. Mosconi et al., "Regional analysis of FDG and PIB-PET images in normal aging, mild cognitive impairment, and Alzheimer's disease," European Journal of
Nuclear Medicine and Molecular Imaging, vol. 35, no. 12, pp. 2169-2181, 2008.

[15] A. Nordberg, "Amyloid plaque imaging in vivo: current achievement and future prospects," European Journal of Nuclear Medicine and Molecular Imaging, vol. 35, no. 1, pp. S46-S50, 2008.

[16] J. Shin, S. Y. Lee, S. H. Kim, Y. B. Kim, and S. J. Cho, "Multitracer PET imaging of amyloid plaques and neurofibrillary tangles in Alzheimer's disease," NeuroImage, vol. 43, no. 2, pp. 236-244, 2008.

[17] S. Aalto, N. M. Scheinin, N. M. Kemppainen et al., "Reproducibility of automated simplified voxel-based analysis of PET amyloid ligand [(11)C]PIB uptake using 30-min scanning data," European Journal of Nuclear Medicine and Molecular Imaging, vol. 36, no. 10, pp. 1651-1660, 2009.

[18] Y. I. Sheline, M. E. Raichle, A. Z. Snyder et al., "Amyloid plaques disrupt resting state default mode network connectivity in cognitively normal elderly," Biological Psychiatry, vol. 67, no. 6, pp. 584-587, 2010.

[19] G. McKhann, D. Drachman, and M. Folstein, "Clinical diagnosis of Alzheimer's disease: report of the NINCDS-ADRDA work group under the auspices of Department of Health and Human Services Task Force on Alzheimer's disease," Neurology, vol. 34, no. 7, pp. 939-944, 1984.

[20] H. C. Padgett, D. G. Schmidt, A. Luxen, G. T. Bida, N. Satyamurthy, and J. R. Barrio, "Computer-controlled radiochemical synthesis: a chemistry process control unit for the automated production of radiochemicals," Applied Radiation and Isotopes, vol. 40, no. 5, pp. 433-445, 1989.

[21] C. A. Mathis, Y. Wang, D. P. Holt, G. F. Huang, M. L. Debnath, and W. E. Klunk, "Synthesis and evaluation of C-labeled 6substituted 2-arylbenzothiazoles as amyloid imaging agents," Journal of Medicinal Chemistry, vol. 46, no. 13, pp. 2740-2754, 2003.

[22] B. J. Lopresti, W. E. Klunk, C. A. Mathis et al., "Simplified quantification of Pittsburgh compound B amyloid imaging PET studies: a comparative analysis," Journal of Nuclear Medicine, vol. 46, no. 12, pp. 1959-1972, 2005.

[23] L. Mosconi, W. H. Tsui, H. Rusinek et al., "Quantitation, regional vulnerability, and kinetic modeling of brain glucose metabolism in mild Alzheimer's disease," European Journal of Nuclear Medicine and Molecular Imaging, vol. 34, no. 9, pp. 1467-1479, 2007.

[24] C. L. Joachim, J. H. Morris, and D. J. Selkoe, "Diffuse senile plaques occur commonly in the cerebellum in Alzheimer's disease," American Journal of Pathology, vol. 135, no. 2, pp. 309-319, 1989.

[25] W. E. Klunk, Y. Wang, G. F. Huang et al., "The binding of 2-(4'-methylaminophenyl)benzothiazole to postmortem brain homogenates is dominated by the amyloid component," Journal of Neuroscience, vol. 23, no. 6, pp. 2086-2092, 2003.

[26] R. P. Woods, S. T. Grafton, J. D. G. Watson, N. L. Sicotte, and J. C. Mazziotta, "Automated image registration: II. Intersubject validation of linear and nonlinear models," Journal of Computer Assisted Tomography, vol. 22, no. 1, pp. 153-165, 1998.

[27] H. Braak and E. Braak, "Neuropathological stageing of Alzheimer-related changes," Acta Neuropathologica, vol. 82, no. 4, pp. 239-259, 1991.

[28] R. M. Nitsch, S. A. Farber, J. H. Growdon, and R. J. Wurtman, "Release of amyloid $\beta$-protein precursor derivatives by electrical depolarization of rat hippocampal slices," Proceedings of the National Academy of Sciences of the United States of America, vol. 90, no. 11, pp. 5191-5193, 1993. 
[29] F. Kamenetz, T. Tomita, H. Hsieh et al., "APP processing and synaptic function," Neuron, vol. 37, no. 6, pp. 925-937, 2003.

[30] J. A. Esteban, "Living with the enemy: a physiological role for the $\beta$-amyloid peptide," Trends in Neurosciences, vol. 27, no. 1, pp. 1-3, 2004.

[31] J. R. Cirrito, K. A. Yamada, M. B. Finn et al., "Synaptic activity regulates interstitial fluid amyloid- $\beta$ levels in vivo," Neuron, vol. 48, no. 6, pp. 913-922, 2005.

[32] E. Abramov, I. Dolev, H. Fogel, G. D. Ciccotosto, E. Ruff, and I. Slutsky, "Amyloid-beta as a positive endogenous regulator of release probability at hippocampal synapses," Nature Neuroscience, vol. 12, no. 12, pp. 1567-1576, 2009.

[33] A. Wree, "Principles of the 2-deoxyglucose method for the determination of the local cerebral glucose utilization," European Journal of Morphology, vol. 28, no. 2-4, pp. 132-138, 1990.

[34] P. J. Magistretti and L. Pellerin, "Cellular mechanisms of brain energy metabolism and their relevance to functional brain imaging," Philosophical Transactions of the Royal Society B, vol. 354, no. 1387, pp. 1155-1163, 1999.

[35] C. P. Chi and E. L. Roberts Jr., "Energy substrates for neurons during neural activity: a critical review of the astrocyte-neuron lactate shuttle hypothesis," Journal of Cerebral Blood Flow and Metabolism, vol. 23, no. 11, pp. 1263-1281, 2003.

[36] S. Ramamurthy and G. V. Ronnett, "Developing a head for energy sensing: AMP-activated protein kinase as a multifunctional metabolic sensor in the brain," Journal of Physiology, vol. 574, no. 1, pp. 85-93, 2006.

[37] S. J. Greco, S. Sarkar, J. M. Johnston, and N. Tezapsidis, "Leptin regulates tau phosphorylation and amyloid through AMPK in neuronal cells," Biochemical and Biophysical Research Communications, vol. 380, no. 1, pp. 98-104, 2009.

[38] G. B. Frisoni, M. Lorenzi, A. Caroli, N. Kemppainen, K. Någren, and J. O. Rinne, "In vivo mapping of amyloid toxicity in Alzheimer's disease," Neurology, vol. 72, no. 17, pp. 15041511, 2009. 


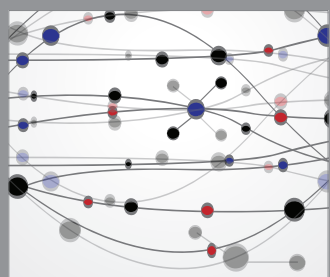

The Scientific World Journal
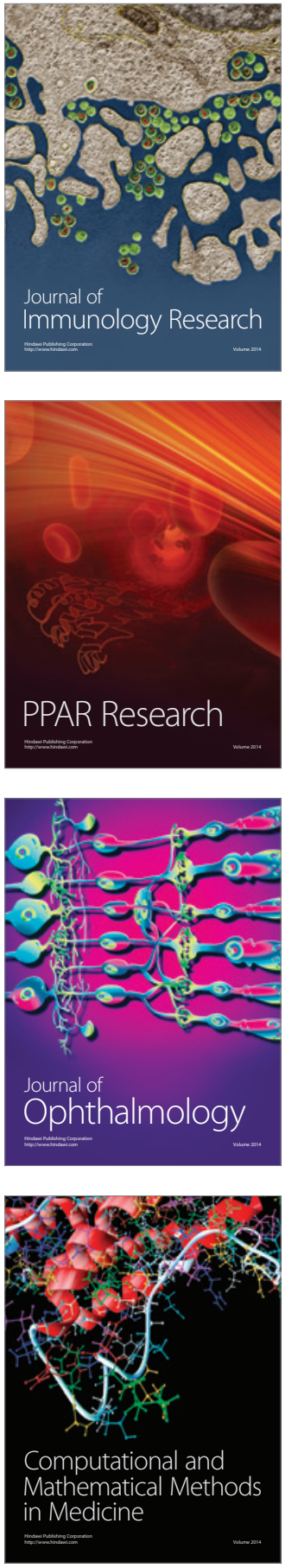

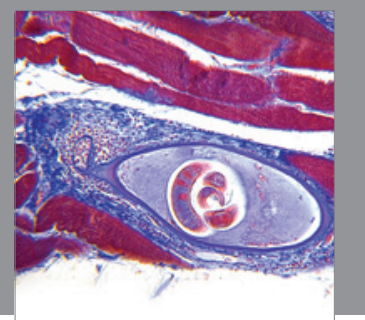

Gastroenterology

Research and Practice
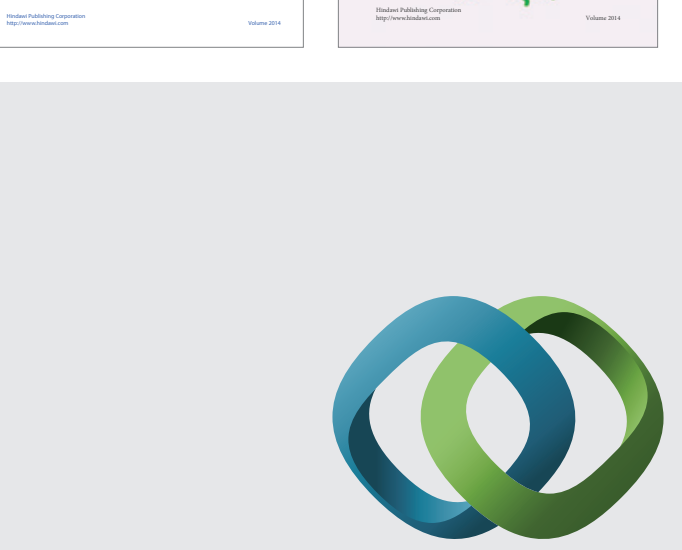

\section{Hindawi}

Submit your manuscripts at

http://www.hindawi.com
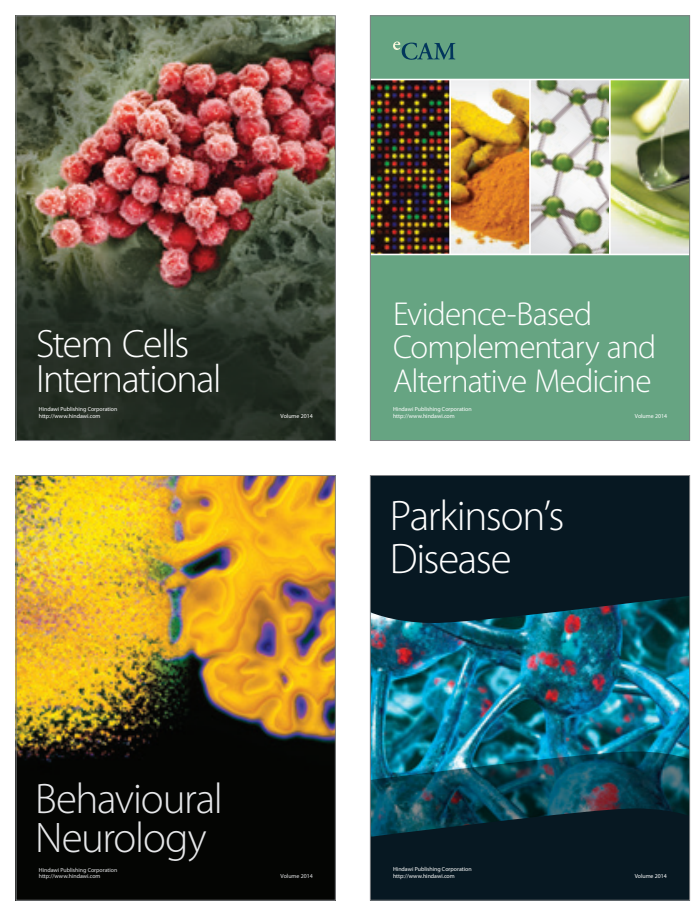

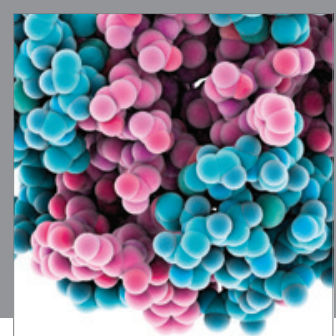

Journal of
Diabetes Research

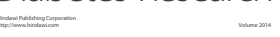

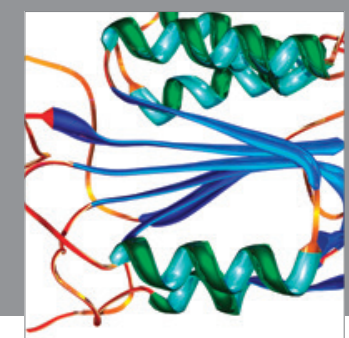

Disease Markers
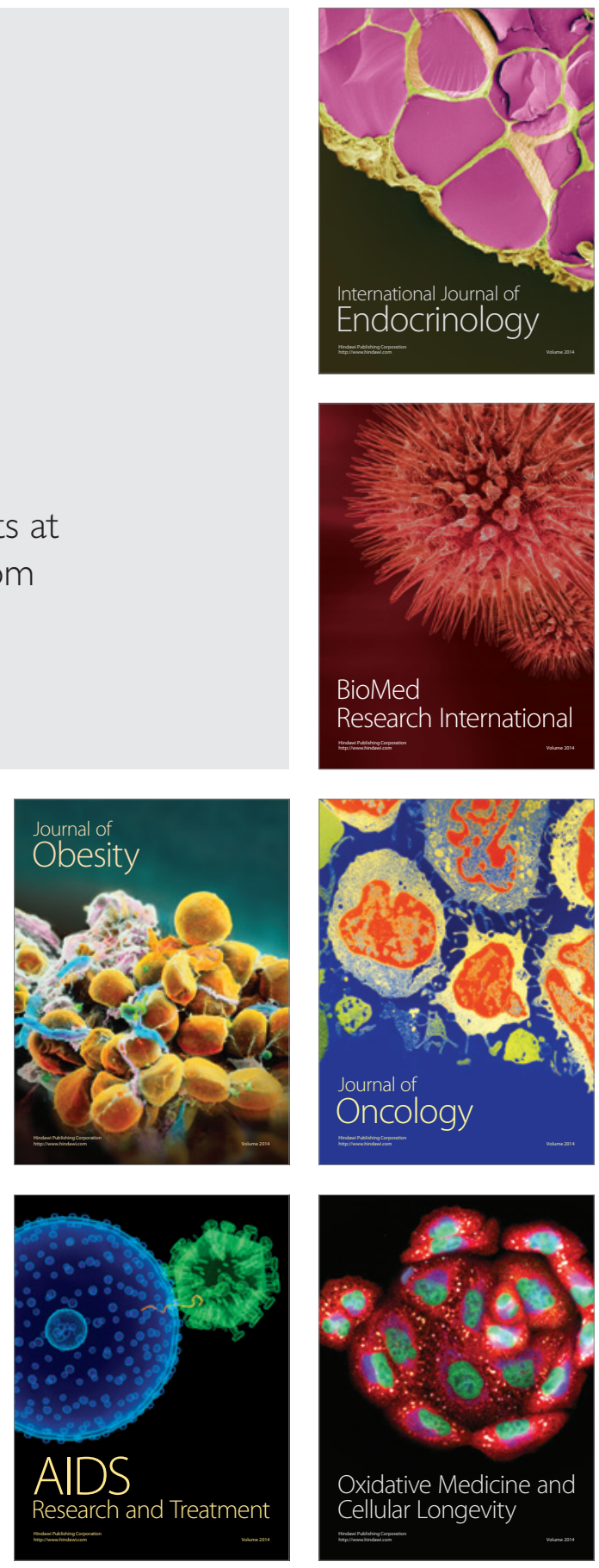